\title{
Philosophische Lebenskunst in der Praxis
}

\author{
W. Schmid
}

\footnotetext{
* Über dieses Spital informiert: Hess C, Hess-Cabalzar A. Menschenmedizin. Von der Vernunft der Vernetzung. Zürich: 2001. Die nachstehenden Ausführungen sind in erweiterter Form in diesem Band enthalten.
}

Korrespondenz:

PD Dr. Wilhelm Schmid

Mindener Strasse 6

D-10589 Berlin

E-Mail: mail@wilhelm-schmid.de
Der Philosophie ist alles zuzutrauen, nur eines nicht: Lebenshilfe. Das ist die Überzeugung der meisten professionellen Philosophen und, so glaube ich, auch vieler Menschen, die die Philosophie in einem Turm aus Elfenbein vermuten. Charakteristisch für alle Philosophie seit Sokrates ist die Frage ti estin: Was ist das? Was liegt dem zugrunde? Was steckt dahinter? Philosophie ist zunächst nichts anderes als ein Innehalten und Nachdenken - das ist eine bescheidene Definition, aber Philosophie beginnt seit jeher mit diesem sokratischen Moment. Menschen haben Fragen an das Leben, und in der Philosophie, nicht in irgendwelchen Fachdisziplinen, ist der rechte Ort, diesen Fragen vorbehaltlos und vorurteilsfrei nachzugehen.

Dass die Philosophie den Raum für grundsätzliche Klärungsprozesse bieten kann, ist zweifellos der Grund für die wachsende Bedeutung der Philosophie in orientierungsloser Zeit. Es geht dabei nicht um eine weitere Form von «Therapie» und auch nicht um eine Bevormundung im Sinne eines normativen «Ratgebens», sondern um die Klärung von Bedingungen, also dessen, «was ist», und darüber hinaus um die Eröffnung von Optionen durch das Denken dessen, «was möglich ist», um zur je eigenen Gestaltung des Lebens und zum Gewinn von Selbstmächtigkeit beizutragen. Auf dieser Grundlage geht es darum, nach dem «guten» und «schönen Leben» zu fragen und ein bewusst geführtes Leben zu ermöglichen. Bewusst geführtes Leben: das ist Lebenskunst.

Aber kann Lebenskunst ein Anliegen der Philosophie sein? Gilt Lebenskunst nicht als ein sehr oberflächlicher Begriff, der das leichte Leben meint: nichts ernst zu nehmen, immer obenauf zu sein? Der Begriff der Lebenskunst entstammt in Wahrheit der antiken Philosophie und ist von Grund auf philosophisch. Philosophie ist Hilfestellung auf dem Weg zur Lebenskunst, und diese Hilfestellung findet vor allem auf der Ebene der nüchternen Analyse und des offenen Denkens statt. In diesem Sinne ist Philosophie Lebenshilfe, und dies seit jeher. Nur die moderne akademische Philosophie hat das im 19. und 20. Jahrhundert aus den Augen verloren, und dafür gab es Gründe: In einer Zeit, in der alle Hoffnungen sich darauf richteten, mit Hilfe von Wissenschaft und Technik sämtliche grossen und kleinen Lebensprobleme lösen zu können, bedurfte es keiner philosophischen Lebenskunst; die Philosophie sah, nicht zuletzt aus vitalen Überlebensgründen, ihre Aufgabe nur noch darin, wissenschaftliche Dienstleistung zu betreiben. Heute dürfte sich das ändern, denn immer deutlicher wird, dass Wissenschaft und Technik zwar einige Lebensprobleme gelöst, neue aber heraufgeführt haben, mit denen viele Menschen kaum mehr zu leben verstehen. So bedarf die Zeit wieder einer Form von Philosophie, die sich den Lebensfragen stellt. Wie dies unter heutigen Bedingungen aussehen kann, lässt sich nur im Experiment erproben. Was meinen Beitrag dazu angeht, versuche ich seit 1998, an einem Spital (jeweils zwei Wochen im September in Affoltern am Albis) einige Arbeit zu leisten.*

Was soll ein Philosoph am Spital? Ich selbst war derjenige, der diese Frage zuallererst stellte, denn ich konnte mir nicht vorstellen, dass die Philosophie in einem Umfeld, in dem es ums ganze Leben geht, sinnvoll sein könnte. Aber die Spitalleitung sah das Potential, das in der Philosophie verborgen liegt, deutlicher. Den Anfang machte ein Essay «Vom Sinn der Schmerzen», den ich 1995 in der Basler Zeitung, dann erneut in dem Buch «Philosophie der Lebenskunst» publizierte. Er zog die Frage nach sich, ob ich nicht auch mal selbst im Krankenhaus arbeiten wolle, um in der Konfrontation mit der Praxis die Idee einer neu zu begründenden Lebenskunst zu erproben. Die Praxis ist für Philosophen allerdings ein Ärgernis. In der Praxis kann die Philosophie scheitern, denn sie tut den Begriffen, die man sich von ihr gebildet hat, selten Genüge, und Philosophen neigen dazu, die Schuld dafür nicht so sehr bei den Begriffen zu suchen, sondern bei der Praxis. Aber was ist eine Lebenskunst wert, die im Leben selbst nichts taugt? So ging ich dorthin, wo die Lebenskunst zweifellos am meisten in Frage steht, erstmals 1998, und dies zu einer Zeit, in der vieles im Gesundheitswesen im Umbruch ist.

Der Philosoph, der von aussen dazukommt, ist mit völlig ungewohnten Erfahrungen konfrontiert und macht sich darüber seine Gedanken, versucht Begriffe dafür zu finden und bestehende Begriffe auf ihre Inhalte hin zu befragen. Was die Situation im Gesundheitswesen angeht, so zeigt sich dem Blick von aussen, dass hier eine exemplarische Diskussion stattfindet. Die 
Gründe für die Probleme liegen vielleicht nur vordergründig in explodierenden Kosten, in Wahrheit dürften sie, wie so vieles, von den Grundstrukturen der Zeit bedingt sein, die es genauer zu betrachten gilt. Welche Antworten auch immer darauf gefunden werden, sie werden wohl zurückwirken auf diese Zeit und nicht etwa nur das Gesundheitswesen verändern.

\section{Allgemeine Situation der Moderne}

Die Zeit, in der wir leben, ist «die Moderne». Moderne, das bedeutet seit zweihundert Jahren, seit dem Zeitalter der Aufklärung, Freiheit, immer grösser werdende Freiheit im Sinne der Befreiung von lästigen Bindungen, demzufolge auch immer umfangreicher werdende Möglichkeiten. Die Triebfeder dieser Freiheit sollten von Anfang an Wissenschaft und Technik sein, angetrieben von einer freien Wirtschaft, die in deren Fortschritt investiert und davon schliesslich wieder profitiert. Das Ziel der auf diese Weise freigesetzten Dynamik sollte «das grösste Glück der grössten Zahl» sein, Glück definiert als «Maximierung von Lust, Eliminierung von Schmerz». Angenehm zu leben, nur angenehm, und alles, was unangenehm ist, mit immer besseren Mitteln auszuschalten, das wird in moderner Zeit zum Traum aller. Und keiner stellt die Frage, ob ein so definiertes Glück überhaupt Sinn hat.

Freiheit ist für die meisten Menschen ein sehr positiv besetzter Begriff. Das Problem ist nur: Freiheit bringt immer auch Kosten mit sich, ideell oder materiell. Die Menschen müssen gerade dann, wenn sie Freiheit erreichen, in aller Freiheit auch Festlegungen treffen, die die Freiheit wieder begrenzen. Sie müssen selbst ihre Haltung und ihr Verhalten wählen und ihr Leben selbst auf bewusste Weise führen, wenn sie wirklich frei leben wollen. Die Gestaltung des Lebens wird somit zur Aufgabe für jeden einzelnen, zur «Autonomie» im Wortsinne der Selbstgesetzgebung. Was einst von Normen bewerkstelligt wurde, muss nun zur Befolgung eines Sollens aus freier Einsicht werden.

Wie kann es zur Einsicht kommen? Nur das Vermögen der Klugheit, das durch eigene Erfahrung und Überlegung wächst, kann wohl leisten, was in früheren Zeiten durch Prinzipien und Normen zustande kam, nämlich vor allem: die Freiheit nicht schrankenlos auszuleben, sondern ihr aus Freiheit Grenzen zu setzen. Dafür bedarf es keiner Moral, auf die kaum noch zu setzen ist, sondern nur einer Klugheit, die auf überlegte Weise den eigenen Interessen dient: Denn das schrankenlose Ausleben der Freiheit fällt letztlich auf den, der im Wortsinne rücksichtslos lebt, selbst zurück, zum Beispiel über wachsende Krankenkassenbeiträge. Daraus kann folgen, aus eigener Einsicht die Ansprüche an eine Perfektionierung der oberflächlich verstandenen «Gesundheit», die immer effizientere und kostenträchtigere Ausschaltung alles Unangenehmen und «Negativen» zu mässigen. Ansonsten führt der immer grösser werdende Anspruch zu immer weiter wachsender Enttäuschung.

\section{Besondere Situation am Spital}

Am Spital Affoltern am Albis wird versucht, neue Wege zu erproben, die Antworten auf die Krise im Gesundheitswesen bereithalten könnten. Die Einbeziehung anderer Disziplinen als nur der Medizin trägt dazu bei. Dieses integrative Konzept wird getragen von einem integrativen Menschenbild, das sowohl den somatischen wie auch den psychischen und geistigen Dimensionen des Menschseins Rechnung tragen kann. Diese doppelte Integrativität ist es, was das singuläre Experiment Affoltern auszeichnet, und sie dient nicht nur auf funktionelle Weise der Institution des Spitals, sondern auf menschliche Weise den Individuen, für die das Spital zum Bezugspunkt wird.

Um das integrative Konzept umzusetzen, kam es frühzeitig zu einer signifikant starken Einbeziehung der Psychotherapie, die nicht nur das medizinische Angebot ergänzt, sondern wesentlich zur Gesprächskultur im ganzen Haus beiträgt. Auf dieser Basis fiel die Entscheidung, neben der traditionellen Einbeziehung der Theologie auch die Philosophie zu beteiligen, um den Lebensfragen der Patienten wie auch der im Spital tätigen Menschen mehr Raum zu geben. Wenn der Versuch, den Menschen als körperlichseelisch-geistige Integrität $\mathrm{zu}$ verstehen, das Anliegen ist, das in Affoltern leitend ist, kann die Philosophie vielleicht dazu beitragen, über die Psychosomatik hinaus zu einer Noopsychosomatik zu kommen, die bei einem integrativen Verständnis des Menschseins auch dem kognitiven Aspekt, der Rolle des Denkens und Nachdenkens, stärker Rechnung trägt.

Die philosophische Arbeit am Spital, die ihre Rolle im Rahmen solcher Überlegungen hat, findet in vierfacher Hinsicht statt:

1. In Vorträgen und Seminaren, in denen einzelne Themen exponiert, dann diskutiert und auf die Praxis am Spital und die jeweils eigene Lebenspraxis bezogen werden. 
2. In Arbeitsgruppen, die eingerichtet werden, um bestimmten Problemen, die sich stellen, intensiv nachzugehen, beispielsweise dem Umgang mit Gewohnheiten, den eigenen und denjenigen von Patienten, oder den epistemologischen Grundlagen eines Gesprächs.

3. In Einzelgesprächen mit Patienten, jedoch auch mit Pflegepersonal und Ärzten, in denen es um eine Lebenssituation oder um Gedanken zum Leben überhaupt gehen kann.

4. In «transversaler Arbeit» - einem Kennenlernen der verschiedensten Abteilungen am Spital durch eine zeitlich begrenzte Mitarbeit, was ermöglichen soll, die wirklich gelebte Praxis möglichst genau kennenzulernen und die integrativen Kräfte am Spital zu stärken.

\section{Philosophische Gespräche}

Vielleicht ist der Philosoph im Krankenhaus ein säkularer Seelsorger, der jedoch die Seelsorge als Anleitung anderer zur Sorge für sich selbst versteht, so dass über die christliche Besetzung des Begriffs hinaus dessen ursprünglich philosophische Bedeutung auf neue Weise wieder Eingang in die Praxis findet. Es ist jedoch klar, dass die Funktion des Philosophen nicht mehr, wie in der Antike, die eines «Seelenarztes» sein kann, der normative Gewissheit darüber hat, wie das Leben zu leben ist. Der Philosoph kann nicht mehr normative, nur optative Funktion haben: Optionen eröffnen, Möglichkeiten aufzeigen, das Für und Wider der verschiedenen Möglichkeiten erörtern, allenfalls einen unverbindlichen Ratschlag aus seiner eigenen Sicht geben, anhand dessen und in Auseinandersetzung damit sein Gegenüber die eigene Position finden und festlegen kann.

Es kann dabei um das Aufzeigen von Gewohnheiten und um deren Reflexion gehen, sowie um das Ausfindigmachen der Strukturen, die Phänomene hervortreiben, die für sich genommen kaum verständlich sind. Schliesslich aber geht es darum, danach zu fragen, was rein denkerisch noch möglich wäre, über das hinaus, was faktisch ist: Vermittlung eines umfassenden Horizonts, in dessen Rahmen das eigene Leben gesehen werden kann. Welche Wahl gibt es oder lässt sich eröffnen, zwischen welchen Alternativen? Welche zwei, drei Möglichkeiten lassen sich ausdenken, welche dieser Möglichkeiten zieht Faszination oder wenigstens Interesse auf sich, und was wäre zu ihrer Verwirklichung wirklich zu tun, welche Organisationsarbeit, welche Gespräche von wem mit wem?

Die Haupttätigkeit des Philosophen am Spital ist, Gespräche zu führen. Wenn Philosophie ein Innehalten und Nachdenken ist, dann ist das Gespräch das gemeinsame Innehalten und Nachdenken, nicht zielführend im Hinblick auf eine zu findende Lösung, sondern als Anregung, das jeweils eigene Denken zu formulieren und sich im Gespräch mit dem Anderen darüber klarer zu werden, alte Anschauungen zu überprüfen und neue Anregungen aufzunehmen. Was geschieht eigentlich in den Gesprächen? Das ist eine entscheidende Frage. Erwartet wird etwas Spektakuläres. Aber es sind in aller Regel unspektakuläre Gespräche. Es ist beinahe unwichtig, was der Inhalt des Gesprächs ist. Das blosse Faktum scheint bereits wichtig zu sein, um zu entlasten, zu ermuntern, anzuregen, etwas zu klären, zu bereinigen, zu befreien ... Der «Trost der Philosophie»: Was viele suchen, ist das Gespräch über das Leben, und zweifellos ist es eine Art «Lebenshilfe», jedoch nicht in einem unmittelbaren Sinn, sondern eher im Sinne sokratischer Geburtshilfe: das ans Tageslicht zu befördern, was im jeweiligen Menschen selbst bereits verborgen liegt; bei der Bewusstwerdung und Formulierung behilflich zu sein.

Der Philosoph «muss» nichts, er erfüllt keine bestimmte Funktion, er ist zu nichts verpflichtet. Vielleicht wird er gerade dadurch als Gesprächspartner interessant. Was zunächst nur meine Verlegenheit war - keinen Plan für die Gesprächsführung zu haben -, erwies sich als Gewinn, um offen zu sein für den Anderen und ihm wirklich zuzuhören, ohne das Gesagte bereits nach bestimmten Erklärungsmustern zu sortieren. «Welchen Plan haben Sie?» eröffnete eine Frau das Gespräch, die bereits sämtliche Formen von Analyse und Therapie durchlaufen hatte und es sich soeben bequem machen wollte, neugierig, mit welchem Muster man ihr diesmal beikommen wolle; sie hatte sich selbst die Rolle der amüsierten Beobachterin zugedacht, wieder «therapieresistent», an der sich eben alle die Zähne ausbeissen, da ihr nicht zu helfen sei: Auch so kann eine «Identität» aussehen. Es wurde ein packendes, irritierendes Gespräch über die Abgründe menschlicher Existenz.

Wichtig ist, dem Gegenüber die Möglichkeit zu bieten, sich über das, was ist und was möglich ist, klarer $\mathrm{zu}$ werden und Plausibilität und Lebenswahrheit zu gewinnen. Wie die Erfahrung zeigt, kann das blosse Gespräch schon Wunder wirken. Das Selbst erfährt im Gespräch die Aufmerksamkeit, die ihm fehlte, die Zuwendung, 
die es entbehrte. Die blosse Aufmerksamkeit eines Anderen kann die Kräfte eines Menschen in ausserordentlichem Masse aktivieren, daher geht es zuweilen darum, nur zuzuhören, stundenlang zuzuhören. Beflügelt womöglich durch die Aufmerksamkeit bietet das Gespräch vor allem einen Anlass zur Selbstaufmerksamkeit. So wird es zum Ereignis, in dem das Selbst von selbst sich wieder findet.

Nichts machen Menschen lieber, als «ihre Geschichte» zu erzählen: das ist die beste Grundlage für das Gespräch. Und das hat Gründe, denn in der Erzählung konstituieren sie sich selbst, das Selbst sucht, findet und konstruiert die Zusammenhänge, die sein Leben durchziehen, und es entscheidet darüber, was davon sein «Inneres», den Kern seiner Kohärenz bilden, was an der Peripherie bleiben soll und was nicht. Was ist Bejahenswertes für sie, was Verneinenswertes? Die dafür erforderliche Hermeneutik der Existenz wird angestossen durch Fragen und findet Anregung im Gespräch, das der Fokus für diese Art von Arbeit sein kann. Indem die Hermeneutik, die Arbeit der Deutung und Interpretation, in Gang kommt, stellt das Selbst die Beziehung zu sich selbst wieder her, die vielleicht verloren oder noch nie so recht gefunden worden war, und leistet damit die entscheidende Arbeit an der eigenen Kohärenz, der Zusammenfügung seiner selbst, seines Lebens und seiner Welt. Diese Zusammenfügung scheint eine entscheidende Ressource der Gesundung zu sein, denn sie erzeugt den «Sinn», der offenkundig unentbehrlich fürs Leben ist.

Aber um die Eigentümlichkeiten der «philosophischen Seelsorge», auch ihre Grenzen, besser kennenzulernen, sie zu verstehen und auf den Begriff zu bringen, wird noch einige Arbeit erforderlich sein.

\section{Wilhelm Schmid}

Freier Philosoph, geb. 1953, lebt in Berlin und lehrt als Privatdozent an der Universität Erfurt und als Gastdozent an der Staatlichen Universität Tiflis (Georgien). Regelmässige Tätigkeit als «philosophischer Seelsorger» am Spital Affoltern am Albis bei Zürich.

\section{Wichtigste Buchpublikationen:}

- Schönes Leben? Einführung in die Lebenskunst. 5. Auflage. Frankfurt/M.: Suhrkamp Verlag; 2002. Übersetzungen: Lettisch (2001), Niederländisch (2001), Serbisch (2001).

- Philosophie der Lebenskunst - Eine Grundlegung. 8. Auflage. Frankfurt/M.: Taschenbuch im Suhrkamp Verlag; 2001. 